\title{
Extreme Values of Wind Speed over the Kara Sea Based on the ERA5 Dataset
}

\author{
Alexander Kislov ${ }^{1}$, Tatyana Matveeva ${ }^{2}$ \\ ${ }^{1}$ Department of Meteorology and Climatology, Faculty of Geography, Lomonosov Moscow State University, Moscow, Russia \\ ${ }^{2}$ Laboratory of Climate, Institute of Geography, Russian Academy of Sciences, Moscow, Russia \\ Email: avkislov@mail.ru,matania.777@gmail.com
}

How to cite this paper: Kislov, A. and Matveeva, T. (2021) Extreme Values of Wind Speed over the Kara Sea Based on the ERA5 Dataset. Atmospheric and Climate Sciences, 11, 98-113.

https://doi.org/10.4236/acs.2021.111007

Received: October 26, 2020

Accepted: January 1, 2021

Published: January 4, 2021

Copyright $\odot 2021$ by author(s) and Scientific Research Publishing Inc. This work is licensed under the Creative Commons Attribution International License (CC BY 4.0).

http://creativecommons.org/licenses/by/4.0/

\section{(c) (i) Open Access}

\begin{abstract}
Extreme values of wind speed were studied based on the highly detailed ERA5 dataset covering the central part of the Kara Sea. Cases in which the ice coverage of the cells exceeded $15 \%$ were filtered. Our study shows that the wind speed extrema obtained from station observations, as well as from modelling results in the framework of mesoscale models, can be divided into two groups according to their probability distribution laws. One group is specifically designated as black swans, with the other referred to as dragons (or dragon-kings). In this study we determined that the data of ERA5 accurately described the swans, but did not fully reproduce extrema related to the dragons; these extrema were identified only in half of ERA5 grid points. Weibull probability distribution function (PDF) parameters were identified in only a quarter of the pixels. The parameters were connected almost deterministically. This converted the Weibull function into a one-parameter dependence. It was not clear whether this uniqueness was a consequence of the features of the calculation algorithm used in ERA5, or whether it was a consequence of a relatively small area being considered, which had the same wind regime. Extremes of wind speed arise as mesoscale features and are associated with hydrodynamic features of the wind flow. If the flow was non-geostrophic and if its trajectory had a substantial curvature, then the extreme velocities were distributed according to a rule similar to the Weibull law.
\end{abstract}

\section{Keywords}

ERA5, Kara Sea, Weibull Probability Distribution Function Wind Speed, Hydrodynamics and Statistics of Extreme Events

\section{Introduction}

A large part of the Kara Sea (Figure 1) is covered with ice year-round. From the 
point of view of temperature, roughness, and other characteristics, this means that the surface of the sea merges with the land. In the warm season, the ice breaks up into separate massifs and the features of the marine surface are manifested in the meteorological regime. The purpose of this article is to study extreme winds above the marine surface when the area of ice covering each grid cell of ERA5 was less than 15\%. At this time of year, an especially strong wind over the Kara Sea is associated with cyclones that make landfall from the west and southwest, and sometimes regenerate over the Kara Sea [1].

The Arctic region is characterized by sparse in-situ observational coverage (conventional coastal weather stations, buoys, ships). Exceedingly few studies (e.g., [2] [3] [4] [5]) have examined climatological Arctic winds from station observations located in the sea-shore zone. Most marine surface wind speed data are provided by satellite sensors (scatterometer, microwave radiometer, altimeter, and synthetic aperture radar) (e.g., [6] [7] [8] [9]). However, sea ice limits satellite use in the Arctic [10], restricting the poleward coverage of the satellite-based characterizations. For example, scatterometer data does not exist for most of the Arctic.

Reanalysis data provide a useful alternative for filling these gaps in wind speed data over the Arctic, as they have global coverage and combine weather forecast models and assimilation of observations from a wide variety of sources. Modelling data (for example, within the framework of the historical CMIP5 experiment) have also been used to assess the pattern of surface wind climatology.

The climatology of winds across the oceans is detailed in multiple works [11] [12] [13], and wind regime information for the Arctic is highlighted in several studies [14] [15]. Regional climatology of near surface winds includes information over Alaska and the adjacent Arctic Ocean [16] [17], over several sectors of Canada [2], over the seas of European and Siberian sectors of Arctic [1] [4], over the northeast Pacific Ocean [5] and so on.

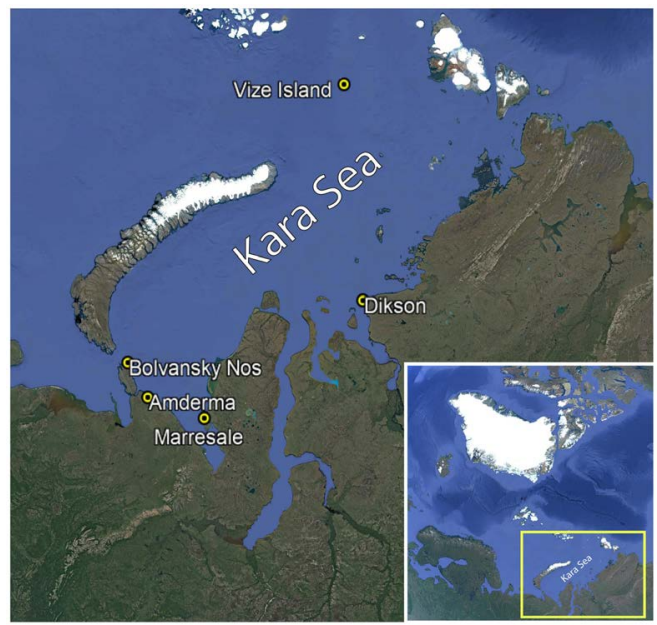

Figure 1. The Kara Sea and locations of observation stations, which were used for ERA5-data comparison. Bottom right insert: map of the Arctic. A yellow frame marks the area for the main map. (Image source: https://www.google.com/maps). 
This study focuses on the Kara Sea, a small part of the Pan-Arctic domain, to more clearly delineate its regional characteristics. For this purpose, a horizontally detailed re-analysis of ERA5 was used. This product (see below) was developed by the European Centre for Medium-Range Weather Forecasts (ECMWF). There is relatively little research that has been published on the climate of this region. Additionally, we consider the issues of hydrodynamic substantiation in evaluating the peculiarity of extreme value statistical laws.

The Weibull distribution has traditionally been used for statistical approximations of wind extremes [4] [11] [18]. The data selected for extreme value analysis must be identically distributed and independent. However, the methods could be used for dependent time series [19] [20]. For an identical distribution, our analysis demonstrated that each set of wind speed extremes (observed in both coastal and open-ocean locations) is a mixture of two different subsets, with each neatly described by the Weibull distribution. The volumes of these subsets are not the same. Almost all samples belong to the so-called base distribution, and only a few percent (or less) of the samples (mostly strong events) are described by another Weibull distribution. Representatives of the base population were marked as swans (and black swans in their upper limit) and representatives of the other group were identified as dragons. This terminology was introduced in several studies [21] [22] [23] [24]. We do not follow these researchers' specifications regarding the details of specific origins of events, their predictability, and so on, and instead use the terms only to mark the differences of samples belonging to various groups.

Apart from the statistical approach, an explanation of the observed wind speed probability distribution should be based on theoretical ideas from hydrodynamic peculiarities of the atmospheric motion. This justification can be obtained by studying the products of numerical simulations or by studying equations that are sufficiently simplified to obtain their analytical solutions. In a previous paper, we concluded that the wind extremes modelled by a general circulation model involved only samples conforming to the base distribution (swans). The same conclusion was derived after reanalysing the ERA Interim dataset. Thus, the numerical coarse resolution products did not contain observed exceptional outliers.

The next step of our analysis was to investigate how accurately a mesoscale atmospheric model (with a fine spatial resolution) simulated the aforementioned peculiarities of wind extremes [5] [25]. We observed that an atmospheric model with a detailed resolution (in this study, we used the data from a domain with a $13.2 \mathrm{~km}$ spatial resolution) did simulate the largest wind speed extremes. Unfortunately, a more thorough analysis showed that the differences in the parameters of the PDFs were still substantial.

In this study, we continue the investigation of the ability of numerical simulations to reproduce wind speed extremes based on the ERA5 dataset [26] [27] [28] also used this approach for multiple simulation types, and ERA5 was marked as the most accurate of the studied group. In [29], the ERA5 surface 
wind data were compared with Advanced Scatterometer (ASCAT) data, and a strong accordance was observed.

Regarding analytical models, several studies (e.g., [30] [31]) have noted that a Rayleigh distribution (a special case of the Weibull distribution) emerges for the wind speed if the vector wind components are assumed to be individually Gaussian. To obtain a physical understanding of the observed PDFs of sea surface wind speeds, a stochastic model for boundary layer winds (including several assumptions and parameterizations) was developed [11]. In our study, we considered another simple hydrodynamics model where a Weibull-like distribution naturally arises for the wind speed anomaly distribution.

In the next section, we describe the data and study area, and briefly summarize the methods. Section 3 describes the evidence for a Weibull distribution in the near surface wind speed. Section 4 is devoted to explaining how the Weibull distribution arises from simplified equations of hydrodynamics. Section 5 concludes the paper.

\section{Data and Methods}

In this study, we used the new global reanalysis ERA5 developed by the ECMWF. The ERA5 reanalysis was improved compared to a previous successful ERA-Interim reanalysis [26]. Specifically, the horizontal resolution was improved to $0.25^{\circ} \times 0.25^{\circ}$, the number of vertical levels was increased to 137 pressure levels from $1000 \mathrm{hPa}$ to $1 \mathrm{hPa}$, the temporal resolution was changed to hourly, and the list of output parameters was extended. Furthermore, the number of assimilated observations was enhanced (approximately five times more compared to ERA-Interim) [27]. Most of the ERA-Interim problems with reprocessing of satellite data were solved and the system of assimilation was improved in ERA5.

For our purposes, we used zonal and meridional components of wind speed at 10 meters, the geopotential at $700 \mathrm{hPa}$ and $850 \mathrm{hPa}$, and the sea ice concentration.

To apply statistical approaches, we composed our data according to the independence condition. Practically, this means that the data sample had to include only independent extreme values. We selected the maximum wind speeds from 3-day intervals in wind speed data for each grid cell. This interval was obtained via autocorrelation function analysis as a period for the disappearance of the correlation between fluctuations (correlation coefficient becomes insignificant). The same time intervals for the same aims were used in several previous studies [4] [32] [33].

During the summer, Kara Sea may either be open water or covered with ice of various concentrations. This causes different roughness conditions, as the roughness of open water is usually lower than that of sea ice. Drag coefficients for open water are approximately 1.5 - 2 times lower than compared to the sea ice surface [34]. Our samples were thus divided into sea ice and open water conditions, because in some regions, the share of days with ice cover reached $40 \%$ - 
$50 \%$. As a criterion for this division, we used the concentration threshold of $15 \%$ (involving in ERA5). The days with a sea ice concentration higher than 15\% were considered as sea ice conditions and lower than $15 \%$ meant open water. We compared statistical results for both open water events and sea ice conditions (see below). In our analysis, we used only open water samples.

\section{Statistical Features of the Observed Sea Surface Wind Speed}

As mentioned, the statistics for extreme wind speed were described by the Weibull distribution. The following equations represent the cumulative distribution function $(W, \mathrm{CDF})$ and the $\operatorname{PDF}(w)$ :

$$
\begin{gathered}
W(u)=1-\exp \left[-\left(\frac{u}{V}\right)^{k}\right] \\
w(u)=\frac{k u^{k-1}}{V^{k}} \exp \left[-\left(\frac{u}{V}\right)^{k}\right]
\end{gathered}
$$

The value of $V$ determines the scale of speed. A value of $u=V$ corresponds to $W(u)=0.63$. This means that $V$ is slightly more than the median $\left(u_{\text {med }}\right)$, and $V=u_{\text {med }} /(\ln 2)^{-1 / k}$. The dependence of moments of the distribution on the Weibull parameters is illustrated in Monahan (2006a). Note that the Weibull distribution for $k=3.6$ approximates a Normal distribution within a range extended to several values of standard deviation.

The Weibull parameters $(k, V)$ are estimated using the maximum likelihood method. One variant of this method is discussed in [4]. In Figure 2, several "Weibull Plots" are shown as calculated based on the ERA5 data, where a straight line is recovered if the sample shows a Weibull distribution. The quality of description we can see visually and quantitatively based on the coefficient of determination $\left(R^{2}\right)$ providing a measure of the success of approximation. At all sites of the Kara Sea, we observed that practically all points of the CDF (besides several points depicting rare and high speeds) showed a close approximation to a Weibull distribution. In a mathematical sense, the use of $\mathrm{R}^{2}$ is related to the application of the Cramer-Mises-Smirnov statistical criterion. The application of the Kolmogorov-Smirnov test also showed that there was no reason not to trust the Weibull distribution (see, for example, [4]).

Thus, most events fit into the basic distribution, and some of the most powerful ones did not fall into it. This result falls under the classification introduced in the Introduction, i.e., when the sample data of the same item refers to different distribution functions. In ERA5, the swans (and black swans) are always represented. Unlike station data, dragons are completely absent in some pixels (Figure 2(a) and Figure 2(b)). This repeats what we observed in results of the general circulation model with a coarse resolution [4] [5]. In other points, they were represented by only a few anomalies that decidedly did not fall within the basic distribution, and it was impossible to estimate distribution parameters 


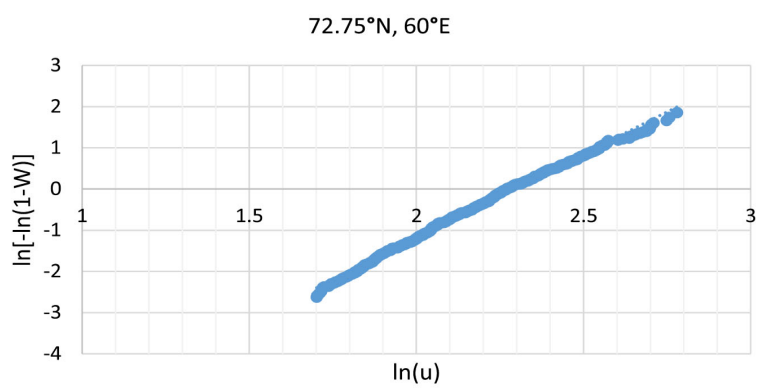

(a)

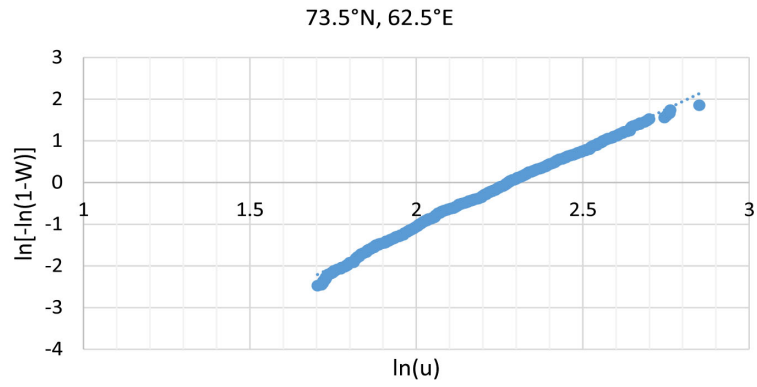

(b)

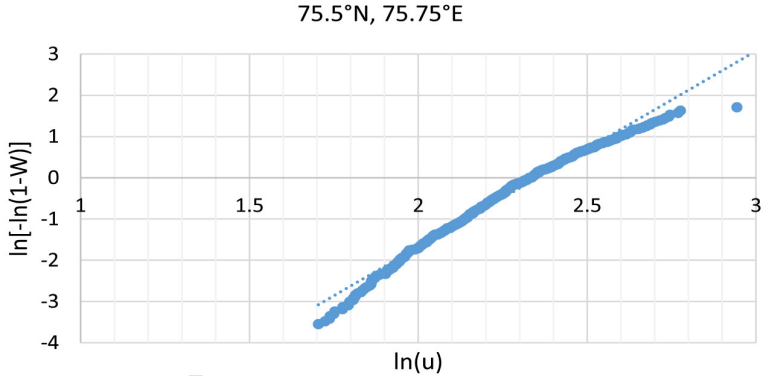

(c)

$71.5^{\circ} \mathrm{N}, 60^{\circ} \mathrm{E}$

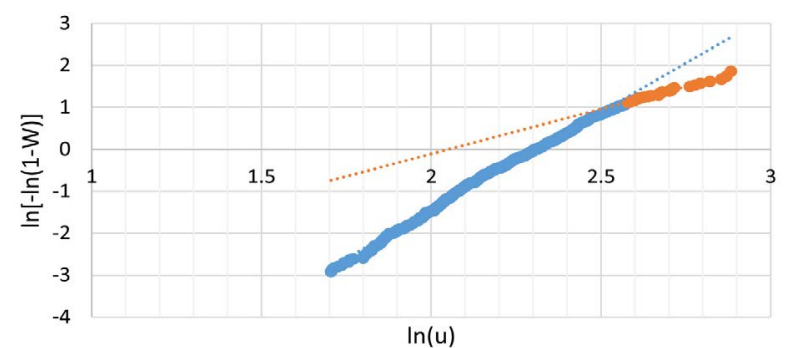

(d)

$71.25^{\circ} \mathrm{N}, 65.5^{\circ} \mathrm{E}$

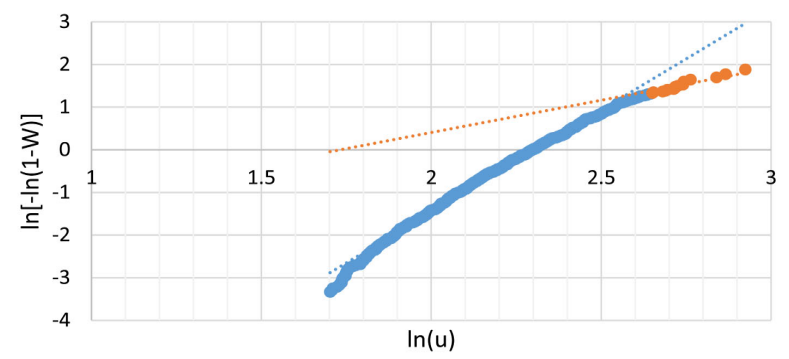

(e) 


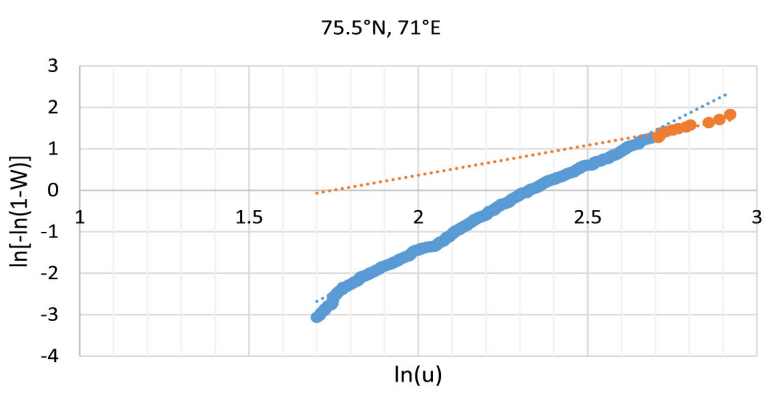

(f)

Figure 2. Cumulative distribution functions of wind speed maxima (ERA5) for 72 hours time step records straightening on the coordinate axis of the Weibull distribution, and linear regression line corresponding to the Weibull function. Examples for different ERA5 grid points ((a)-(f)).

from such a small volume of samples (Figure $2(\mathrm{c})$ ). In some pixels, a sufficient number of linearly spaced points dropped out of the base distribution, which emphasized their commonality and belonging to the same distribution law (Figures 2(d)-(f)). Therefore, we considered (based on statistical criteria) that the estimation of the distribution parameters was acceptable. As a result, said estimation was implemented for 126 pixels (out of 520 covering the studied area).

As a rule, with respect to a certain group of points, it is impossible to determine the population that they belong to, as the trend lines on the graphs practically coincide (Figure 2). In this case, we attributed them both to the swans and dragons.

In the basic distribution, the values of $V$ and $k$ were unique in different pixels, but $V$ varied minimally, i.e., from 9.5 to $10.5 \mathrm{~m} / \mathrm{s}$. Changes in the exponent were much more substantial (from 3 to 5). The parameter $k$ increased in the south and east directions of the region, adjacent to Novaya Zemlya (Figure 3). In the central part of the Kara Sea, $k$ was close to 3.6. Here, the probability distribution was close to Gaussian. Further south and east, the $k$ increased and the tail of the PDF became lighter than that of the Gaussian distribution, which meant that the probability of strong winds continuously lessened until unrealistically small values were reached. However, this conclusion will fundamentally change when the presence of dragons is considered. Therefore, we can conclude that the geographical features of the PDFs were determined by changes in the exponent. Having expressed the moments of distribution through $V$ and $k$ [11], we observed that the skewness was near zero (from -0.2 to +0.2 ), and the kurtosis varied from -0.03 to -0.3 .

For dragons, the exponent in the Weibull distribution was substantially less than for swans (the value of $\mathrm{k}$ varied from 1 to 3 ). This meant that the distribution differed from the normal distribution because of the presence of a heavier tail, and that the likelihood of strong winds increased.

In Figure 4, all results are summarized in the parameter field $(k, V)$. Each population had its own range of values, and a clear connection of parameters was present. 


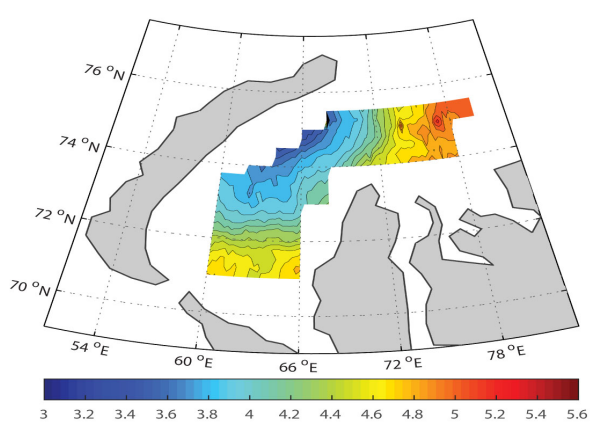

Figure 3. Spatial distribution of the Weibull parameter $k$.

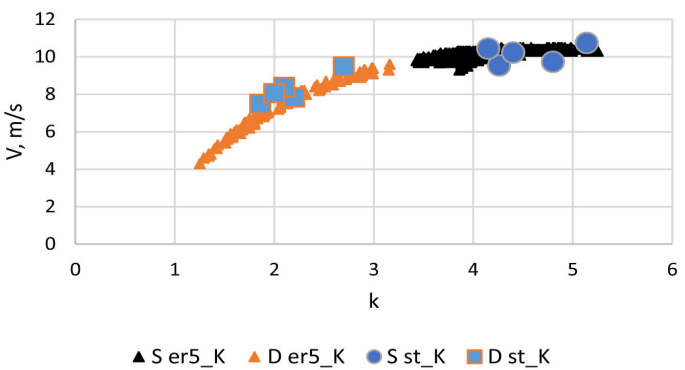

Figure 4. The Weibull distribution parameters $(k$ and $V)$ calculated for all pixels of the ERA5 (covering central part of the Kara Sea-see Figure 3), S er5_K and D er5_K for swans and dragons, respectively, and additionally, data from stations located around the Kara Sea (see Figure 1), S st_K and D st_K for swans and dragons, respectively.

The expression for the basic range, i.e., the swans, was given by $V=0.36 k+8.7$ with a low coefficient of determination. As $V$ varied minimally compared to the variations of $k$, and there was thus no reason to expect a correlation. For dragons, $V=5.62 \ln (k)+3.29, R^{2}=0.99$. A close relationship between the parameters meant that the Weibull distribution encompassed a single parameter.

The reason for this unambiguity was unclear; it may have been a consequence of the algorithm for calculating the wind speed near the sea surface in the ERA5 reanalysis. Alternately, this may have occurred because we examined a relatively small area, as it contained a uniform wind regime. A comparison of the parameters $(k, V)$ according to station measurements in the Arctic does not demonstrate such a close relationship. There was an increase in $V$ with increasing $k$. Existence of a strong connection between $V$ and $k$ was not noted according to the scatterometer [11].

Figure 4 includes similar data on measurements at five stations located in the coastal zone of the Kara Sea: Bolvansky Nos $\left(70.5^{\circ} \mathrm{N}, 59.1^{\circ} \mathrm{E}\right)$, Amderma $\left(69.8^{\circ} \mathrm{N}, 61.7^{\circ} \mathrm{E}\right)$, Marresale $\left(69.7^{\circ} \mathrm{N}, 66.8^{\circ} \mathrm{E}\right)$, Vize Island $\left(79.5^{\circ} \mathrm{N}, 77.0^{\circ} \mathrm{E}\right)$, and Dikson $\left(73.5^{\circ} \mathrm{N}, 80.2^{\circ} \mathrm{E}\right)$ [4]. These data are in accordance with the reanalysis data, thus emphasizing its high quality.

Even though anomalies related to dragons are rare events, their presence or absence are unprincipled for the parameters of the basic distribution, and neglecting them can lead to an incorrect interpretation of the results. To illustrate, consider the situation at pixel $71.5^{\circ} \mathrm{N}, 60^{\circ} \mathrm{E}$. In this cell, there were 26 events re- 
lated to "dragons" (with respect to 20 events, it was impossible to make a conclusion about which affiliation, i.e., dragons or black swans, that they belonged to) (Figure 2(d)). The Weibull distribution parameters were $k=4.66$ and $V=$ $10.1 \mathrm{~m} / \mathrm{s}$ for swans, and $k=2.14$ and $V=7.8 \mathrm{~m} / \mathrm{s}$ for dragons. If we estimate the average $\bar{u}$ and the variance $D$ from the base distribution (using well-known formulas: $\bar{u}=V \Gamma(1+1 / k), \quad D=V^{2} \Gamma(1+2 / k)-\bar{u}^{2}, \Gamma$ is the gamma function), we obtain $\bar{u}=9.2 \mathrm{~m} / \mathrm{s}$ и $D=6.15 \mathrm{~m}^{2} / \mathrm{s}^{2}$. If at this point the sample is not divided into swans and dragons, then $k=4.46$ and $V=10.2 \mathrm{~m} / \mathrm{s}$ and $и$ $\bar{u}=9.3 \mathrm{~m} / \mathrm{s}$ и $D=6.1 \mathrm{~m}^{2} / \mathrm{s}^{2}$. The distribution moments are almost identical in the two examples; however, without isolating the dragon population, the largest anomalies are outside the scope of statistical analysis. Accordingly, the quantile value corresponding to a probability of 0.99 , as calculated from the base distribution, was $u_{0.99}=V[-\ln 0.01]^{1 / k}=14 \mathrm{~m} / \mathrm{s}$. According to the distribution of dragons, we then obtain $u_{0.99}=16 \mathrm{~m} / \mathrm{s}$. Naturally, this result demonstrates that in reality extrema occur much more often than is prescribed by the basic distribution.

Figure 5 compares pairs of quantile values for the pixels in which, in addition to swans, it was possible to identify dragons and evaluate the parameters of their distribution. Similar data on station measurements are given here; interestingly, $u_{0.99}$ was larger for dragons. Station data were characterized by large differences between representatives of different populations. This result, together with the already noted situation that the statistical properties of dragons were evaluated only in a quarter of cases, suggested that the ERA5 did not fully provide information on the largest extremes. We encountered the same phenomenon when analysing COSMO-CLM data with a horizontal step of $13.2 \mathrm{~km}$ : The model reproduced dragons, but they were not as powerful as those obtained from the measurement data [5].

Consider what happens if the selection of information (on the grounds of a lack of ice cover) is not carried out. The calculations showed, first, that with ice, the wind speed distribution was described by the Weibull distribution with a high accuracy (the determination coefficients never fell below 0.95). Second,

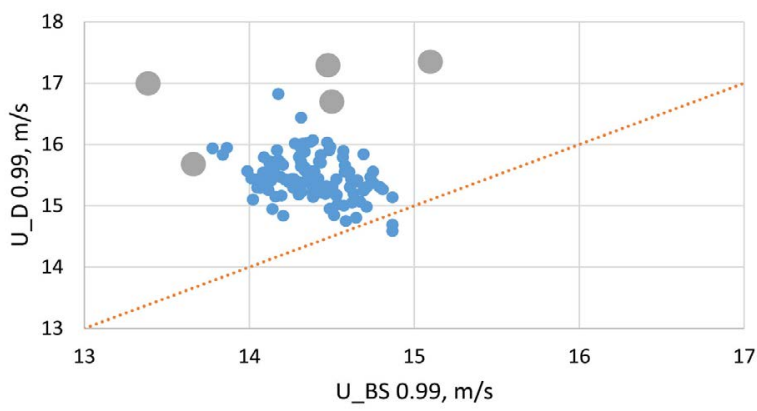

Figure 5. Quantile wind speed values $U(0.99)$ in $\mathrm{m} / \mathrm{s}$ for wind data from ERA5 calculated separately for two groups of wind speed extremes come from the black swans and dragons populations, and additionally, data from stations located around the Kara Sea (see Figure 1). 
dragons were almost completely absent in the sample. Third, the exponent over an open surface was always greater than approximately $15 \%$, and the magnitude of $\mathrm{V}$ is greater by $10 \%$. As a result, the average value from the set of extrema was less by $1 \mathrm{~m} / \mathrm{s}$ above the ice, and the variance was greater by $1 \mathrm{~m}^{2} / \mathrm{s}^{2}$. It is possible that the surface roughness was responsible for this effect.

\section{Applicability of the Weibull Distribution for the PDF of Wind Speed}

The purpose of this section is to understand why the probabilities of extreme velocities were described by the Weibull distribution.

From a probabilistic point of view, the applicability of the Weibull distribution for extreme value analysis is generally based on the following concept. Starting with a parent distribution whose CDF is $Q(U)$, the distribution is sampled $m$ times, and the maximum value of the $m$ samples is obtained. This maximum value has a CDF of simply $Q^{m}$. Next, knowing the shape of the initial distribution, we can proceed to the law for extreme values. This allows them to be fit to one of three limiting distributions [35] [36]. One type of limiting distributions is the Weibull distribution, which has traditionally been used for statistical approximations of wind speed extremes.

On the other hand, it is clear that the probability distribution of anomalies should be determined by the flow hydrodynamics. Accordingly, research has demonstrated that detailed numerical products such as the ERA5 or those derived from mesoscale models are capable of reproducing the observed statistical features of the wind regime within the main features. Conversely, coarse-resolution models only reproduce anomalies related to the base distribution.

To obtain a physical understanding of the observed and simulated PDFs of surface wind speeds, we consider the simple hydrodynamic model. This model should reflect the behaviour of the velocity modulus, as this value is used in statistical studies. The determination of an analytical justification for the Weibull type distribution law was attempted based on the characteristics of the hydrodynamic flow.

For this aim, following the classical book on the subject [37], we consider the natural coordinate system. This system is defined by the orthogonal set of unit vectors $\boldsymbol{s}$ (oriented parallel to the horizontal velocity at each point) and $\boldsymbol{n}$ (normal to the horizontal velocity). The dynamics of the horizontal momentum are determined by the following equations:

$$
\begin{gathered}
\frac{\mathrm{d} U}{\mathrm{~d} t}=-g \frac{\partial H}{\partial s} \\
\frac{U^{2}}{R}+f U=-g \frac{\partial H}{\partial n}
\end{gathered}
$$

For our task, the analysis of these equations is mostly suitable because $U$ denotes the horizontal speed as a nonnegative scalar. $R$ is the curvature radius, and $f$ is the Coriolis parameter. For a stationary case when the motion is parallel to 
the geopotential line $g \partial H / \partial s=0$, and dynamics are determined in Equation (3). We consider cyclonic motion (which corresponds to the conditions $R>0$, $\partial H / \partial n<0$ ), as under these synoptic conditions the greatest anomalies of wind speed are achieved. We also consider the curvature and the Coriolis parameter to be constant values on a certain segment of the trajectory.

Viscosity and the effect of friction are not included in Equation (3). However, this does not preclude analysis, as it can be assumed that the flow is considered outside the atmospheric boundary layer. For the task of studying near-surface wind, this is not a limitation, because maximum velocities are associated with the transfer of large momentum values from the lower troposphere to the surface [38] [39]. Concurrently, stationarity, the absence of both vertical movements and the influence of the latent heat realization in situ, deprives the model of several important effects. In part, these effects are reflected in the curvature of the flow, but in any case, this is only an indirect characterization.

Because the geostrophic wind is defined as $U_{g}=-(g / f) \partial H / \partial n$, Equation (3) is transformed by:

$$
\frac{U^{2}}{R f}+U=U_{g}
$$

Equation (4) can be used to calculate the PDF of the wind speed through knowing the PDF of the geostrophic wind. The latter can be estimated by considering the PDF of the geopotential height.

For this purpose, we calculated the PDFs of the variations of the geopotential height at 850 and $700 \mathrm{hPa}$ pressure levels for individual grid cells of the study area based on ERA5 data. These levels above the boundary layer were chosen because we analysed motion without friction (see above). The PDFs had the characteristic shape of Gaussian curves (bell curve) (Figure 6). We used one-sample Kolmogorov-Smirnov and Jarque-Bera tests to verify a match of a normal distribution to data samples; these tests showed a goodness of fit of a normal distribution at the $5 \%$ significance level.

We then considered the difference in geopotential heights at points " 1 " and "2". The difference of $H_{1}-H_{2} \equiv \delta H$ determines the geostrophic wind. The determination of the density function of the sum (difference) of two quantities with a normal distribution is a classical problem of probability theory. As a result, the PDF is given simply by the Gaussian curve

$$
r(\delta H)=\frac{1}{2 \sigma \sqrt{\pi(1-\rho)}} \exp \left[-\frac{\delta H^{2}}{4 \sigma^{2}(1-\rho)}\right]
$$

Here, $\sigma$ is standard deviation of the height, and $\rho$ is the autocorrelation coefficient between height fluctuations at point " 1 " and " 2 ".

Alternately, we can replace this expression for the PDF of geostrophic winds:

$$
q\left(U_{g}\right)=\frac{1}{2 \sigma_{g} \sqrt{\pi(1-\rho)}} \exp \left[-\frac{U_{g}^{2}}{4 \sigma_{g}^{2}(1-\rho)}\right]
$$

Here, $\sigma_{g}$ is standard deviation of the geostrophic wind. 


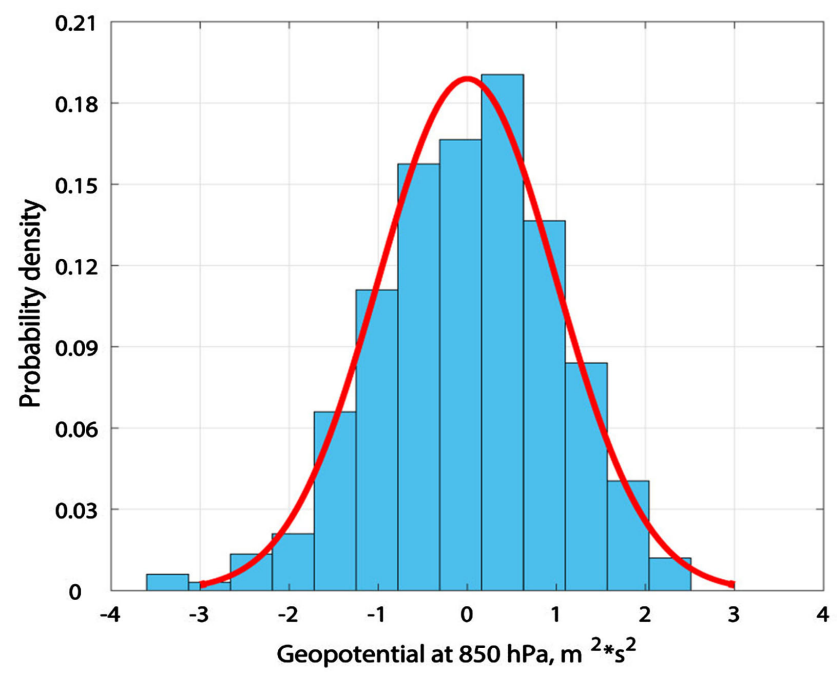

(a)

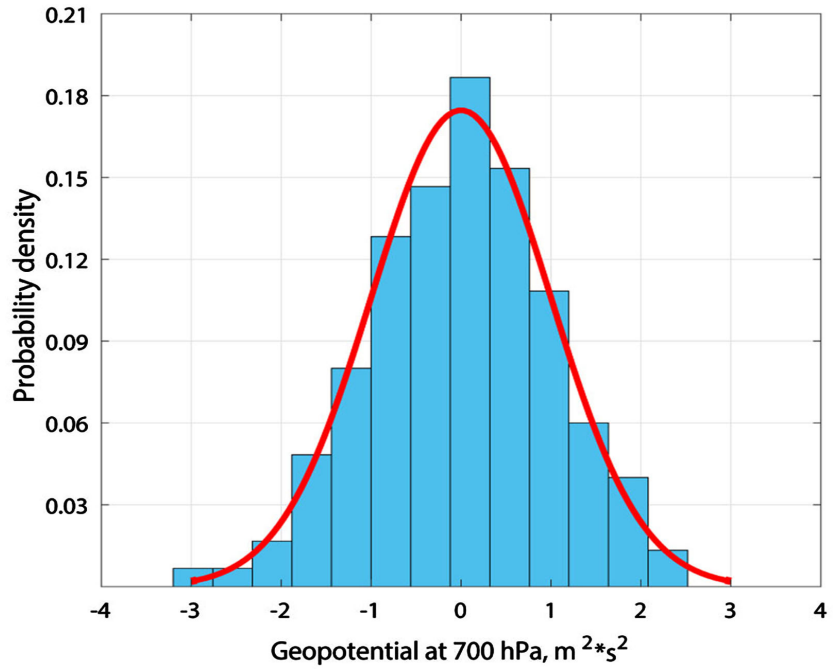

(b)

Figure 6. Cumulative distribution functions calculated for time series of geopotential height (at 850 (a) and 700 (b) hPa levels) together with Gaussian curves. Kolmogorov-Smirnov Test statistic $($ KS-stat $)=0.0346(\mathrm{a}),($ KS-stat $)=0.0261(\mathrm{~b})$.

In considering Equation (4) and the function $q\left(U_{g}\right)$ (6), the CDF of wind velocity is given by:

$$
G(U)=\int_{0}^{U_{g}} q(x) \mathrm{d} x=\int_{0}^{U^{2} / R f+U} q(x) \mathrm{d} x
$$

The PDF calculates as:

$$
\begin{gathered}
\dot{G}(U) \equiv g(U)=q\left(\frac{U^{2}}{R f}+U\right) \cdot\left[\frac{2 U}{R f}+1\right] \\
g(U)=\left(\frac{2 U}{R f}+1\right) \cdot \frac{1}{2 \sigma_{g} \sqrt{\pi(1-\rho)}} \cdot \exp [\Psi] \\
\Psi=-\left(U^{2} / R f+U\right)^{2} /\left[4 \sigma_{g}^{2}(1-\rho)\right]
\end{gathered}
$$


A complex combination of functions resembles the Weibull distribution. The exponent depends on the curvature of the trajectory. When $R f$ is approximately $10 \mathrm{~m} / \mathrm{s}$, the effective degree is 3.5 ; at $R f=\sim 1 \mathrm{~m} / \mathrm{s}$, the degree is already 3.9, and at $R f=\sim 0.1 \mathrm{~m} / \mathrm{s}$, the effective degree is practically 4 . These results are in accordance with those obtained according to EPA5 (see Figure 3 ) and other identical results, although the entire range of changes in $k$ was not covered.

Comparing Expressions (9) and (1b), the similarity of their overall structures can be observed, although the factor in front of the exponent is not the same as required (see (1b)). The theory is suitable only for a basic distribution and can serve as an explanation of the probability of the appearance of black swans. The transition from black swans to dragons in the framework of this approach is not reproduced; for this purpose, apparently, we must consider the factors that in this case remained out of sight (see above). The plausibility of this thesis is indicated by our finding that, as already noted, dragons along with black swans were found in the results of reproduction of the wind by the mesoscale model. However, despite certain shortcomings, this result (depicted by the Equation (9)) can be considered successful. This is because, generally, we confirmed that in a stationary flow, the distribution of velocity anomalies was determined by a Weibull type distribution.

\section{Conclusions}

The data were analysed on ice-free (with ice coverage less than $15 \%$ of the cell area) cells of the Kara Sea. In many pixels, the extreme wind speed sample ERA5 was split into swans (and black swans) and dragons. In a quarter of the grid nodes examined, the parameters of the Weibull probability distribution function could be estimated not only for the swan sample, but also for the dragon population. The practical importance of highlighting dragons was that the largest anomalies are skipped without the former's presence. It is easy to create these errors in the automatic processing of information without special controls.

For swans and dragons, such a close relationship was found between parameters of the Weibull distribution to the point where it subsequently was classified as a one-parameter distribution. It remains unclear whether this uniqueness of the connection was a consequence of the features of the calculation algorithm used in the ERA5, or whether it was a consequence of the relatively small water area, with close conditions for the formation of anomalies, that was considered.

The manifestation of the general laws of extreme velocity statistics is predetermined by the general hydrodynamic peculiarities of flow. The curvature of the flow played a key role in distinguishing these peculiarities from the normal distribution of wind speed anomalies. As expected, ruggedness of the trajectory associated with non-geostrophic movements in mesoscale systems was reflected in extreme velocities. We were able to show that the distribution function of the anomalies had a shape close to that of the Weibull distribution. This demonstrates the bridge between the hydrodynamics and statistics of extreme events. 


\section{Acknowledgements}

This work has received funding from the Russian Foundation for Basic Research (RFBR) (project number 18-05-60147) and Lomonosov Moscow State University (grant AAAA-A16-116032810086-4).

\section{Data Availability Statement}

General data used in this study is archived in the repository (Datasets Generated: "Mendeley Data", https://data.mendeley.com/datasets/vdy2nksk4h/1).

\section{Conflicts of Interest}

The authors declare no conflicts of interest regarding the publication of this paper.

\section{References}

[1] Kislov, A. and Matveeva, T. (2020) The Monsoon over the Barents Sea and Kara Sea. Atmospheric and Climate Sciences, 10, 339-356. https://doi.org/10.4236/acs.2020.103019

[2] Hundecha, Y., St-Hilaire, A., Ouarda, T.B.M.J., El Adlouni, S. and Gachon, P. (2008) A Nonstationary Extreme Value Analysis for the Assessment of Changes in Extreme Annual Wind Speed over the Gulf of St. Lawrence, Canada. Journal of Applied Meteorology and Climatology, 47, 2745-2759.

[3] Wan, H., Wang, X.L. and Swail, V.R. (2010) Homogenization and Trend Analysis of Canadian Near-Surface Wind Speeds. Journal of Climate, 23, 1209-1225. https://doi.org/10.1175/2009JCLI3200.1

[4] Kislov, A. and Matveeva, T. (2016) An Extreme Value Analysis of Wind Speed over the European and Siberian Parts of Arctic Region. Atmospheric and Climate Sciences, 6, 205-223. http://dx.doi.org/10.4236/acs.2016.62018

[5] Kislov, A. and Platonov, V. (2019) Analysis of Observed and Modelled Near-Surface Wind Extremes over the Sub-Arctic Northeast Pacific. Atmospheric and Climate Sciences, 9, 146-158. https://doi.org/10.4236/acs.2019.91010

[6] Zhang, H.M., Bates, J.J., Reynolds, R.W. (2006) Assessment of Composite Global Sampling: Sea Surface Wind Speed. Geophysical Research Letters, 33, Article ID: L17714. https://doi.org/10.1029/2006GL027086

[7] Sampe, T. and Xie, S.-P. (2007) Mapping High Sea Winds from Space: A Global Climatology. Bulletin of the American Meteorological Society, 88, 1965-1978. https://doi.org/10.1175/BAMS-88-12-1965

[8] Young, I.R., Zieger, S. and Babanin, A.V. (2011) Global Trends in Wind Speed and Wave Height. Science, 332, 451-455. https://doi.org/10.1126/science.1197219

[9] Zieger, S., Babanin, A.V. and Young, I.R. (2014) Changes in Ocean Surface Wind with a Focus on Trends in Regional and Monthly Mean Values. Deep Sea Research Part I: Oceanographic Research Papers, 86, 56-67. https://doi.org/10.1016/j.dsr.2014.01.004

[10] Hullinger, W.J. and Long, D.G. (2014) Mitigation of Sea Ice Contamination in QuikSCAT Wind Retrieval. IEEE Transactions on Geoscience and Remote Sensing, 52, 2149-2158. https://doi.org/10.1109/TGRS.2013.2258400

[11] Monahan, A.H. (2006a) The Probability Distribution of Sea Surface Wind Speeds. 
Part I: Theory and Sea Winds Observations. Journal of Climate, 19, 497-520. https://doi.org/10.1175/JCLI3640.1

[12] Monahan, A.H. (2006b) The Probability Distribution of Sea Surface Wind Speeds Part II: Dataset Intercomparison and Seasonal Variability. Journal of Climate, 19, 521-534. https://doi.org/10.1175/JCLI3641.1

[13] Stopa, J.E., Cheung, K.F., Tolman, H.L. and Chawla A. (2013) Patterns and Cycles in the Climate Forecast System Reanalysis Wind and Wave Data. Ocean Modelling, 70, 207-220. https://doi.org/10.1016/j.ocemod.2012.10.005

[14] Hughes, M. and Cassano, J.J. (2015) The Climatological Distribution of Extreme Arctic Winds and Implications for Ocean and Sea Ice Processes. Journal of Geophysical Research: Atmospheres, 120, 7358-7377. https://doi.org/10.1002/2015JD023189

[15] Surkova, G. and Krylov, A. (2019) Extremely Strong Winds and Weather Patterns over Arctic Seas. Geography, Environment, Sustainability, 12, 34-42. https://doi.org/10.24057/2071-9388-2019-22

[16] Stegall, S.T. and Zhang, J. (2012) Wind Field Climatology, Changes, and Extremes in the Chukchi-Beaufort Seas and Alaska North Slope during 1979-2009. Journal of Climate, 25, 8075-8089. https://doi.org/10.1175/JCLI-D-11-00532.1

[17] Redilla, K., Pearl, S.T., Bieniek, P.A. and Walsh, J.E. (2019) Wind Climatology for Alaska: Historical and Future. Atmospheric and Climate Sciences, 9, 683-702. https://doi.org/10.4236/acs.2019.94042

[18] Palutikof, J.P., Brabson, B.B., Lister, D.H. and Adcock, S.T. (1999) A Review of Methods to Calculate Extreme Wind Speeds. Meteorological Applications, 6, 119-132. https://doi.org/10.1017/S1350482799001103

[19] Beirlant, J., Goegebeur, Y., Segers, J., Jozef Teugels, J., De Waal, D. and Ferro, C. (2004) Statistics of Extremes: Theory and Applications. Willey Series in Probability and Statistics, John Wiley \& Sons Ltd., Chichester. https://doi.org/10.1002/0470012382

[20] Coles, S. (2001) An Introduction to Statistical Modeling of Extreme Values. Springer Series in Statistics. Springer-Verlag, London. https://doi.org/10.1007/978-1-4471-3675-0

[21] Taleb, N.N. (2010) The Black Swan: The Impact of the Highly Improbable. 2nd Edition, Penguin, New York.

[22] Sornette, D. (2009) Dragon-Kings, Black Swans and the Prediction of Crises. Research Paper No. 09-36, Swiss Finance Institute, Zürich. http://dx.doi.org/10.2139/ssrn.1470006

[23] Sornette, D. and Ouillon, G. (2012) Dragon-Kings: Mechanisms, Statistical Methods and Empirical Evidence. The European Physical Journal Special Topics, 205, 1-26. https://doi.org/10.1140/epjst/e2012-01559-5

[24] Wheatley, S. and Sornette, D. (2015) Multiple Outlier Detection in Samples with Exponential \& Pareto Tails: Redeeming the Inward Approach \& Detecting Dragon Kings. Research Paper No. 15-28, Swiss Finance Institute, Zürich. https://doi.org/10.2139/ssrn.2645709

[25] Kislov, A., Rivin, G., Platonov, V., Varentsov, M.I., Rozinkina, I.A., Nikitin, M.A. and Chumakov, M.M. (2018) Mesoscale Atmospheric Modelling of Extreme Velocities over the Sea of Okhotsk and Sakhalin. Izvestiya, Atmospheric and Oceanic Physics, 54, 322-326. https://doi.org/10.1134/S0001433818040242

[26] Hersbach, H. and Dee, D. (2016) ERA-5 Reanalysis Is in Production. ECMWF 
Newsletter, No. 147.

[27] Hersbach, H., Bell, B., Berrisford, P., Horányi, A., Sabater, J.M., Nicolas, J., Radu, R., Schepers, D., Simmons, A., Soci, C. and Dee, D. (2019) Global Reanalysis: Goodbye ERA-Interim, Hello ERA5. ECMWF Newsletter, 159, 17-24.

[28] Ramon, J., Lledó, L., Torralba, V., Soret, A. and Doblas-Reyes, F.J. (2019) What Global Reanalysis Best Represents Near-Surface Winds? Quarterly Journal of the Royal Meteorological Society, 145, 3236-3251. https://doi.org/10.1002/qj.3616

[29] Rivas, M.B. and Stoffelen, A. (2019) Characterizing ERA-Interim and ERA5 Surface Wind Biases Using ASCAT. Ocean Science, 15, 831-852. https://doi.org/10.5194/os-15-831-2019

[30] Meissner, T., Smith, D. and Wentz F. (2001) A 10 Year Intercomparison between Collocated Special Sensor Microwave Imager Oceanic Surface Wind Speed Retrievals and Global Analyses. Journal of Geophysical Research, 106, 11731-11742. https://doi.org/10.1029/1999JC000098

[31] Cakmur, R., Miller, R. and Torres, O. (2004) Incorporating the Effect of Small-Scale Circulations upon Dust Emission in an Atmospheric General Circulation Model. Journal of Geophysical Research, 109, Article ID: D07201. https://doi.org/10.1029/2003JD004067

[32] Coles, S.G. and Walshaw, D. (1994) Directional Modelling of Extreme Wind Speeds. Journal of the Royal Statistical Society. Series C (Applied Statistics), 43, 139-157. https://doi.org/10.2307/2986118

[33] Gusella, V. (1991) Estimation of Extreme Winds from Short-Term Records. Journal Struct. Engineering, 117, 375-390. https://doi.org/10.1061/(ASCE)0733-9445(1991)117:2(375)

[34] Mai, S., Wamser, C. and Kottmeier, C. (1996) Geometric and Aerodynamic Roughness of Sea Ice. Boundary-Layer Meteorology, 77, 233-248. https://doi.org/10.1007/BF00123526

[35] Fisher, R.A. and Tippett, L.H.C. (1928) Limiting Forms of the Frequency Distribution of the Largest or Smallest Members of a Sample. Mathematical Proceedings of the Cambridge Philosophical Society, 24, 180-190. https://doi.org/10.1017/S0305004100015681

[36] Gnedenko, B. (1943) Sur la Distribution limite du Terme Maximum D’unesériealéatoire. Annals of Mathematics, 44, 423-453. (In French) https://doi.org/10.2307/1968974

[37] Holton, J.R. and Hakim, G.J. (2013) An Introduction to Dynamic Meteorology. 5th Edition, Academic Press, Cambridge, 552 p. https://doi.org/10.1016/C2009-0-63394-8

[38] Brasseur, O. (2001) Development and Application of a Physical Approach to Estimating Wind Gusts. Monthly Weather Review, 129, 5-25. https://doi.org/10.1175/1520-0493(2001)129<0005:DAAOAP>2.0.CO;2

[39] Schulz, J.-P. and Heise, E. (2003) A New Scheme for Diagnosting Near-Surface Convective Gusts. COSMO Newsletter, 3, 221-225. 